\title{
Impact of Saturation of Fatty Acids of Phosphatidylcholine and Oil Phase on Properties of $\beta$-Lactoglobulin at the Oil/Water Interface
}

\author{
Theresia Heiden-Hecht $^{1}$ D . Stephan Drusch ${ }^{1}$
}

Received: 8 April 2021 / Accepted: 13 November 2021 / Published online: 9 December 2021

(c) The Author(s) 2021

\begin{abstract}
Oil in water emulsions are commonly stabilized by emulsifying constituents like proteins and/or low molecular weight emulsifiers. The emulsifying constituents can compete or coexist at the interface. Interfacial properties thus depend on molecular structure of the emulsifying constituents and the oil phase and the resulting molecular interactions. The present study systematically analyzed the impact of fatty acid saturation of triacylglycerides and phosphatidylcholine on the interfacial properties of a $\beta$-lactoglobulin-stabilized interface. The long-term adsorption behaviour and the viscoelasticity of $\beta$-lactoglobulin-films were analyzed with or without addition of phosphatidylcholine via drop tensiometry and dilatational rheology. Results from the present study showed that increasing similarity in fatty acid saturation and thus interaction of phosphatidylcholine and oil phase increased the interfacial tension for the phosphatidylcholine alone or in combination with $\beta$-lactoglobulin. The characteristics and stability of interfacial films with $\beta$-lactoglobulin-phosphatidylcholine are further affected by interfacial adsorption during changes in interfacial area and crystallization events of low molecular weight emulsifiers. This knowledge gives guidance for improving physical stability of protein-based emulsions in foods and related areas.
\end{abstract}

Keywords Emulsifier $\cdot$ Protein $\cdot$ Interactions $\cdot$ Rheology $\cdot$ Emulsion $\cdot$ Crystallization

\section{Introduction}

Oil in water emulsions are common systems in the food or pharmaceutical industry. The immiscible phases are stabilized with emulsifying constituents namely proteins and/or low molecular weight emulsifiers. Both types of emulsifying constituents have the ability to decrease the interfacial tension of the system whereby their stabilizing mechanism

\section{Highlights}

- Interfacial tension is affected by interactions of phospholipids with the oil phase

- Similar effects occur when phospholipids and protein coexist at the interface

- Viscoelastic behaviour of the interfacial film depends on molecular interactions

- Major reasons are differences in solubility and crystallization effects

Theresia Heiden-Hecht

t.heiden-hecht@tu-berlin.de

1 Faculty III Process Sciences, Institute of Food Technology and Food Chemistry, Department of Food Technology and Food Material Science, Technische Universität Berlin, Straße des 17. Juni 135, 10623 Berlin, Germany at the oil/water interface is rather different [1]. Proteins are dissolved in the aqueous phase. The molecular structure of the proteins is determined by their amino acid sequence and the folding of the protein [2]. They adsorb at the interface, unfold and form a viscoelastic layer with several inter- and intramolecular interactions [3, 4]. An interfacial layer with high viscoelasticity is favourable concerning the stability of emulsions against coalescence $[1,5]$. In comparison, depending on their molecular structure low molecular weight emulsifiers can be dissolved in the aqueous phase or in the oil phase $[6,7]$. Their molecular structure consists of a hydrophilic head group like e.g. phosphatidylcholine and fatty acids with different degree of saturation and chain length [8]. They have a high interfacial activity and stabilize emulsions or foams based on the Gibbs-Marangoni mechanism and/or weak electrostatic interactions [1,9].

In a wide range of applications, proteins and low molecular weight emulsifiers are actively used in combination or co-occur through their presence in specific food ingredients. The molecular structure of both emulsifying constituents defines their interactions and interfacial arrangement. In general, proteins can be displaced by highly interfacial active low molecular weight emulsifiers $[1,10]$ or both can coexist 
at the interface. Coexistence can result in interactions via hydrogen bonding, hydrophobic or electrostatic effects [7, $11,12]$. Electrostatic effects depend on the isoelectric point of the proteins [2], the $\mathrm{pk}_{\mathrm{a}}$ value for low molecular weight emulsifiers [13] and the characteristics of the aqueous phase like $\mathrm{pH}$ or ionic strength [2]. The multitude of interactions between emulsifying constituents determines the characteristics and viscoelasticity of interfacial films and thus emulsion stability. A film with low viscoelasticity is a result of a low number of, repulsive or hydrophobic interactions [1, 9]. In contrast, a film with high viscoelasticity results from attractive interactions [9].

The characteristics of a mixed interfacial film with proteins and low molecular weight emulsifiers also depend on the interactions with the lipophilic phase. Variables affecting these interactions are the chemical nature of the lipophilic phase, the structure of the protein as well as the head group and the type of fatty acids in the hydrophobic tail of the low molecular weight emulsifier. Although different lipophilic phase have already been used to study the interfacial properties of dairy proteins [14-17], no systematic investigation on the impact of interactions between proteins and oil phase on interfacial properties exists. In other recent studies, Bergfreund et al. investigated the impact of alkanes or alkane substituents as lipophilic phases on interfacial tension and viscoelasticity of several protein sources or the interactions between lipophilic phases and surfactants with the same hydrophobic tail but different head group [18-20]. To the best of our knowledge, systematic studies on low molecular weight emulsifiers with similar head group, but different fatty acid chains and different oil phases are not available.

In general, interactions between lipophilic components comprise dispersion forces or $\pi$-interactions of double bindings [21, 22]. The interaction of the low molecular weight emulsifiers and the oil phase are stronger with increasing similarity in fatty acid chain length and saturation as well as solubility of the emulsifier in the lipophilic phase [23]. Since saturated fatty acids have a linear fatty acid chain and unsaturated fatty acids have a kinked chain with an angle of $40^{\circ}$ per double bond [22], interactions between unsaturated and saturated fatty acids are not strong and hindered by steric issues. As a consequence, the interfacial occupation and arrangement can be influenced by the interactions between the lipophilic constituents. A recent study from Hildebrandt et al. (2016) showed that strong interactions between lipophilic phase and low molecular weight emulsifier increase the concentration required to reach a monolayer concentration at the interface [24]. This study investigated phosphatidylcholine with unsaturated or saturated fatty acids in a lipophilic phase with double bindings [24]. Finally, the structure of the low molecular weight emulsifiers and oil phase affect the crystallization behaviour and morphology of the lipophilic components as well as their interactions.
Depending on the phase transition behaviour, low molecular weight emulsifiers may form a template for heterogenous nucleation at the interface [23]. A crystallized low molecular weight emulsifier may be detected with an increase in interfacial viscoelasticity, even if proteins like casein or whey protein are present [25-27].

Therefore, the aim of the present study is to determine the impact of lipophilic interactions of phosphatidylcholine and oil phase in dependence on saturation of fatty acid chains on the interfacial properties of mixed interfacial films. It is hypothesized that the interfacial tension is higher for a phosphatidylcholine with increasing interactions with the oil phase. At low concentrations, the interfacial tension of a $\beta$-lactoglobulin-phosphatidylcholine stabilized interface is decreased due to coexistence of the emulsifying constituents at the interface. An interfacial film stabilized with $\beta$-lactoglobulin-phosphatidylcholine reacts more elastic during expansion and compression if the phosphatidylcholines are not strongly interacting with the oil phase. This effect is caused by a higher concentration of phosphatidylcholines at the interface, faster stabilization of unoccupied interfacial regions or a solid template at the interface depending on saturation of fatty acids of phosphatidylcholines.

To fulfil the aim, we investigated two phosphatidylcholine samples differing in the fatty acid composition, but similar in their head group and two oils differing in fatty acid composition. The development of interfacial tension was measured with or without addition of phosphatidylcholines via drop tensiometry in a long-term time range. The viscoelasticity of the corresponding interfacial films was investigated using dilatational rheology.

\section{Materials and Methods}

$\beta$-lactoglobulin was isolated from whey protein isolate (Bipro, Agropur Dairy Cooperative Inc. Minnesota, USA) with a method described elsewhere [28, 29]. The resulting protein powder has a dry matter content of $92.8 \pm 1.2 \%$ and a protein content of $92.8 \pm 0.4 \%$. The protein fraction consists of $99.6 \%$ isolated $\beta$-lactoglobulin and $0.4 \% \alpha$-Lactalbumin which was analyzed according to [28]. Medium chain triglyceride oil (MCT-oil) WITARIX® MCT 60/40 was kindly provided from IOI Oleo GmbH (Hamburg, Germany). Sunflower seed oil from Helianthus annuus was purchased from Sigma Aldrich Chemie GmbH (Steinheim, Germany). The MCT-oil consists of $0.1 \%$ C6:0, 56.4\% C8:0, 43.3\% $\mathrm{C} 10: 0$ and $0.1 \% \mathrm{C} 12: 0$ and has an acid value of $0.04 \%$. The sunflower oil consists of $6 \% \mathrm{C} 16: 0,4 \% \mathrm{C} 18: 0,26 \% \mathrm{C} 18: 1$ and $63 \% \mathrm{C} 18: 2$ and has an acid value of $0.09 \%$. Both oils were treated with magnesium silicate (Florisil@ from Carl Roth $\mathrm{GmbH}$, Karlsruhe, Germany) to remove interfacial active substances. The interfacial tension was controlled 
to be constant via drop tensiometry. After purification, the sunflower oil was immediately frozen in portions and was thawed for each experiment individually. Two phosphatidylcholines were kindly provided from Lipoid $\mathrm{GmbH}$ (Ludwigshafen, Germany). Phospholipon $® 90 \mathrm{H}$ consists of molecules with $96.9 \%$ phosphatidylcholine as head group with fatty acids of $98 \%$ of C16:0 and C18:0, 0.1\% C18:1 and C18:2. Lipoid S100 consists of 97.4\% phosphatidylcholine as head group with fatty acids of $12-17 \% \mathrm{C} 16: 0,2-5 \%$ C18:0, 7-12\% C18:1, 59-70\% C18:2 and 5-8\% C18:3. Both phosphatidylcholines are obtained from soy origin.

\section{Preparation of Protein Solutions}

For adsorption behaviour and dilatational rheology measurement, $\beta$-lactoglobulin was dissolved and stirred in distilled water for about $2 \mathrm{~h}$. The $\mathrm{pH}$ of the $0.01 \mathrm{~d} . \mathrm{m} . \%$ $\beta$-lactoglobulin solution was adjusted to $\mathrm{pH} 7$ with $0.1 \mathrm{M}$ and $1 \mathrm{M} \mathrm{NaOH}$. The solutions were stored at $5{ }^{\circ} \mathrm{C}$ for about $14 \mathrm{~h}$ overnight. Afterwards, the solutions were stirred, and the $\mathrm{pH}$ was adjusted if necessary.

\section{Preparation of Phosphatidylcholine-Oil Solutions}

The phosphatidylcholines were dissolved in MCT-oil or sunflower oil at a concentration of $0.1 \%$. These oil-phosphatidylcholine solutions were used as stock solution and were heated to the melting of the respective phosphatidylcholines. The choice of the heat treatment was made according to the specification sheet and visual observation as shown in Fig. 1. $90 \mathrm{H}$-solutions were heated to $90{ }^{\circ} \mathrm{C}$ for $15 \mathrm{~min}$ and $\mathrm{S} 100$ solutions were heated to $50{ }^{\circ} \mathrm{C}$ for $15 \mathrm{~min}$. The heat-treated stock solutions were diluted to investigate the adsorption behaviour of phosphatidylcholines alone or in combination with $\beta$-lactoglobulin.

\section{Long Term Adsorption Behaviour of Phosphatidylcholines with or without $\beta$-Lactoglobulin}

Long term adsorption behaviour was measured by drop tensiometry (PAT1M, Sinterface Technologies e.K., Berlin. Germany). The drop shape is recorded with a high-speed camera and the interfacial tension is calculated from the drop curvature with the help of the improved Young Laplace equation. The droplet size was chosen at $30 \mathrm{~mm}^{3}$.

Concentration series of phosphatidylcholines were recorded from $0.1 \%, 0.01 \%, 0.001 \%, 0.0005 \%$ to $0.0001 \%$. The diluted phosphatidylcholine-oil solutions were heated to $90{ }^{\circ} \mathrm{C}$ for 8 min before measurement to ensure melting of phosphatidylcholines. A droplet of distilled water was formed in MCT-oil-phosphatidylcholine solutions at $60.5 \pm 1.5{ }^{\circ} \mathrm{C}$. The interfacial tension was recorded for $30 \mathrm{~min}$ including the $10 \mathrm{~min}$ cooling period to room temperature. In Fig. 1, representative MCT-oil-phosphatidylcholine solutions are shown. For all concentrations, the measurement with visual equipment like a drop tensiometer is feasible. Only the investigation of the stock solution with $0.1 \%$ $90 \mathrm{H}$ is not feasible, if the temperature approaches a value of $50{ }^{\circ} \mathrm{C}$ or below. This solution developed a high turbidity caused by crystallization.

The characteristics of interfacial films with phosphatidylcholines and $\beta$-lactoglobulin were measured with one chosen phosphatidylcholine concentration of $0.0001 \%$. Therefore, a $0.01 \% \beta$-lactoglobulin droplet was formed in a MCT-oil or sunflower-oil-phosphatidylcholine mixture. The
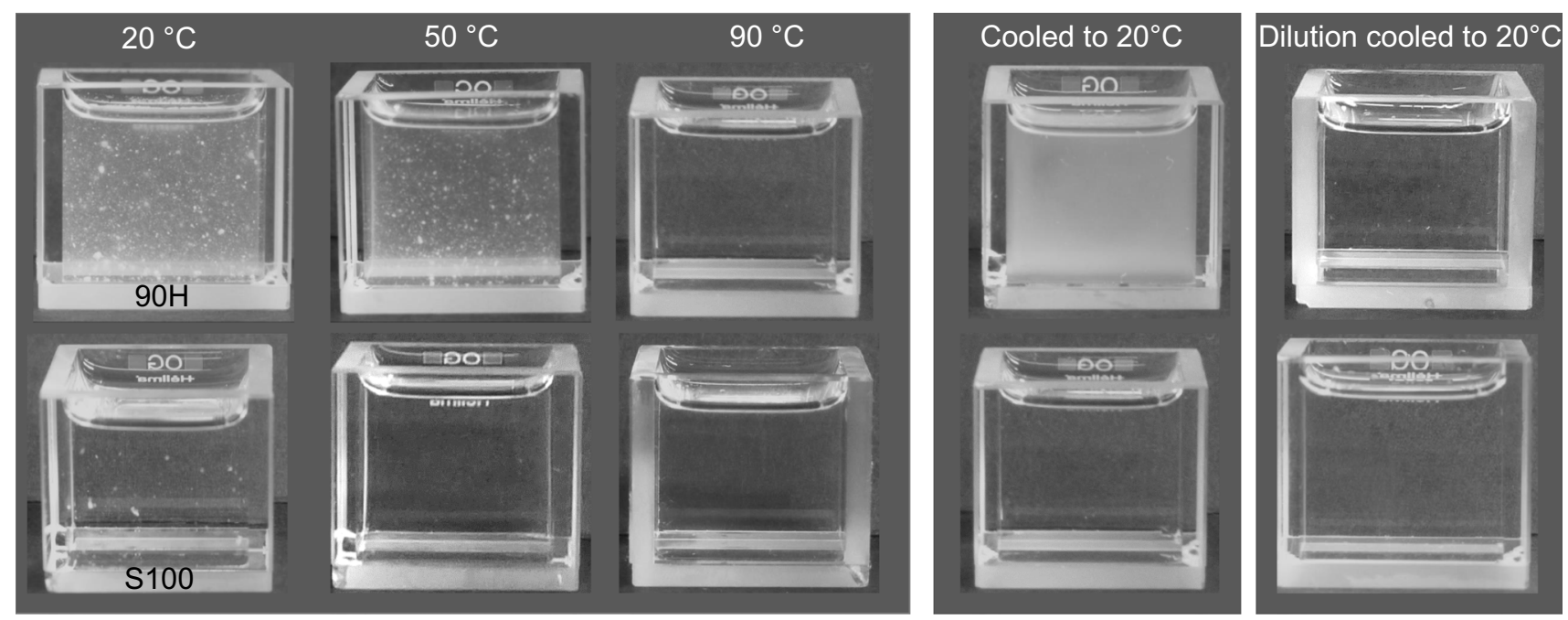

Fig. 1 Images of solutions with $0.1 \%$ phosphatidylcholines (S100 or $90 \mathrm{H}$ ) in MCT-oil during heating to $90{ }^{\circ} \mathrm{C}$ and subsequent cooling to $20{ }^{\circ} \mathrm{C}$. Diluted phosphatidylcholine-MCT-oil solutions with a concentration of $0.0001 \%$ cooled to $20^{\circ} \mathrm{C}$. 
phosphatidylcholine-oil heat and cooling treatment was the same as described above whereby the interfacial tension was recorded for $3 \mathrm{~h}$. For long term adsorption behaviour, a method standard deviation was calculated based on five replicated measurements of a representative $\beta$-lactoglobulinphosphatidylcholine sample.

\section{Dilatational Rheology}

Dilatational rheology was investigated by pendant drop tensiometry (PAT1M, Sinterface Technologies e.K., Berlin, Germany). A high-speed camera was used to record the change in drop shape during sinusoidal oscillation.

A $\beta$-lactoglobulin droplet was formed in MCT-oil or sunflower-oil-phosphatidylcholine mixtures. The drop was equilibrated for $3 \mathrm{~h}$ (see long term adsorption behaviour). Subsequently, a frequency sweep ( $2 \%$ amplitude, $0.002 \mathrm{~Hz}$ to $0.1 \mathrm{~Hz})$ followed by an amplitude sweep $(0.01 \mathrm{~Hz}, 1 \%$ to $5 \%$ amplitude) were performed.

The complex dilatational modulus $\left(\mathrm{E}^{*}\right)$ is calculated from the proportion of the change in interfacial tension $(\sigma)$ and area (A) (eq.1) [30]. The elastic modulus (E') and the viscous modulus (E") are determined with the help of eq.2. The phase angle $(\phi)$ is calculated with $\tan (\phi)=E$ "'E' [30]. A phase angle of $0^{\circ}$ represents entirely elastic behaviour and a phase angle of $90^{\circ}$ represents entirely viscous behaviour. A value between $0^{\circ}$ and $90^{\circ}$ is attributed to viscoelastic behaviour of the film.

$E^{*}=\frac{d \sigma}{d \ln A}$

$E^{*}=E_{d}+i \omega \eta_{d}=E^{\prime}+i E^{\prime \prime}$

Beside the described dilatational moduli and phase angle, the data of dilatational rheology is presented with Lissajousplots. These figures show the change in interfacial tension $\left(\Delta \mathrm{IFT}=\sigma-\sigma_{0}\right)$ versus the change in area $\left(\Delta \mathrm{A} / \mathrm{A}_{0} ; \Delta \mathrm{A}=\mathrm{A}\right.$ $\left.-\mathrm{A}_{0}\right) . \sigma_{0}$ and $\mathrm{A}_{0}$ represent the interfacial tension and area at zero strain. For dilatational rheology, a method standard deviation was calculated based on five replicated measurements of a representative sample.

\section{Statistical Analysis}

Statistical analysis was performed by analysis of variance (ANOVA). Significance was measured by post-hoc Scheffé test $(\mathrm{p}<0.05)$ using IBM SPSS statistics version 28.0.0.0 (IBM Corp., Armonk, USA). Statistical results are displayed in Fig. 3 and 4, and in Fig. 5 and 7 for interfacial tension and elastic moduli, respectively. One may pay attention when discussing the outcome of the statistical analysis. It is based on the typical standard deviation of the methodology as determined from multiple measurements of a representative sample from these experiments, but not individual independent measurements of each sample.

\section{Results and Discussion}

\section{Long Term Adsorption Behaviour of Phosphatidylcholines in MCT-Oil}

The interfacial tension of the pure oil-water interface amounted to $25.4 \pm 0.5 \mathrm{mN} / \mathrm{m}$. High concentrations of
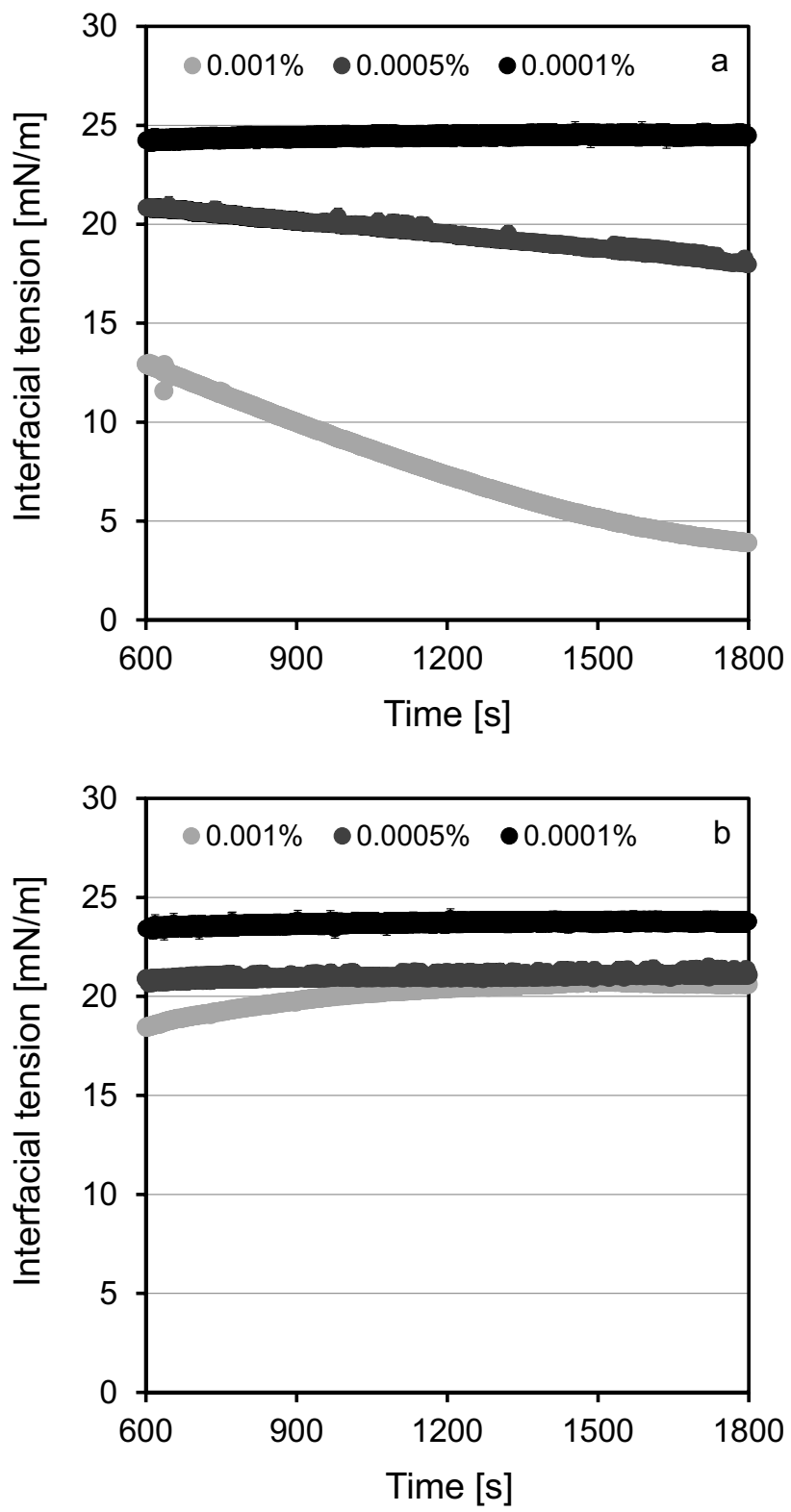

Fig. 2 Interfacial tension of concentration series of phosphatidylcholines $\mathrm{S} 100$ (a) and 90H (b) in MCT-oil in a concentration range from 0.001 to $0.0001 \%$ at room temperature. Error bars display the coefficient of variation of the method. 


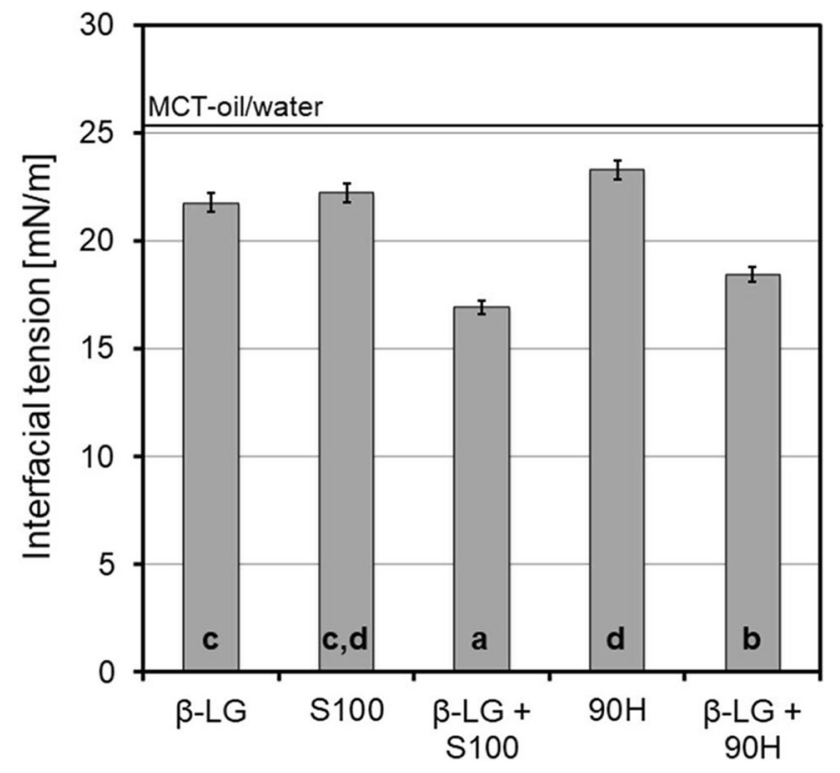

Fig. 3 Interfacial tension of $0.01 \% \beta$-lactoglobulin, $0.0001 \%$ phosphatidylcholines $90 \mathrm{H}$ and S100 in MCT-oil alone or in combination after $3 \mathrm{~h}$ droplet ripening time. Error bars display the coefficient of variation of the method, letters a-d indicate significant differences $(\mathrm{p}<0.05)$.

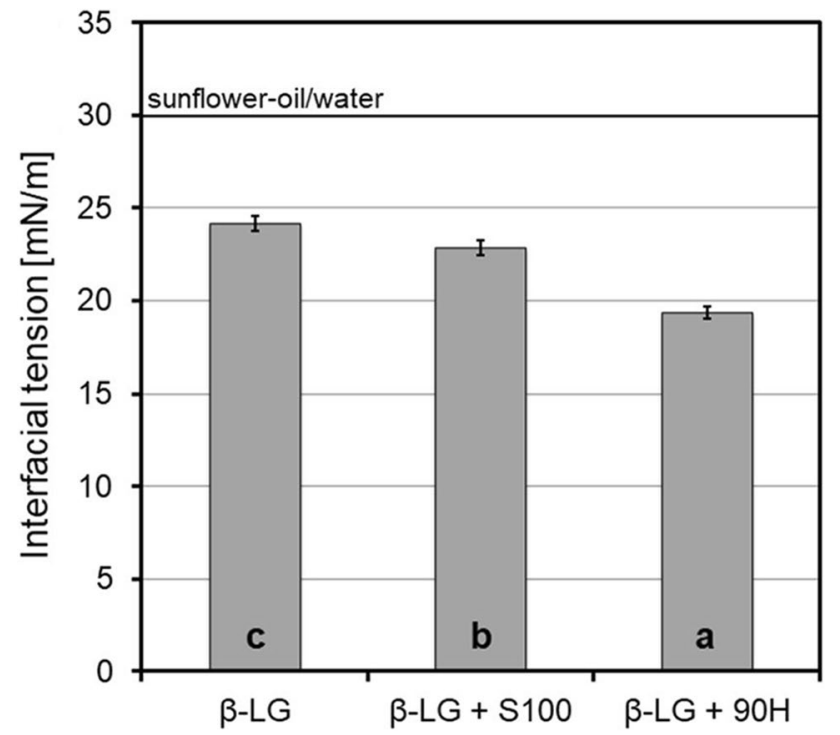

Fig. 4 Interfacial tension of $0.01 \% \beta$-lactoglobulin alone or in combination with $0.0001 \%$ phosphatidylcholines $90 \mathrm{H}$ and $\mathrm{S} 100$ in sunflower-oil after $3 \mathrm{~h}$ droplet ripening time. Error bars display the coefficient of variation of the method, letters a-c indicate significant differences $(\mathrm{p}<0.05)$.

$0.1 \%$ and $0.01 \%$ of the low molecular weight emulsifiers were not analyzed, since the droplets detached from the needle during the measurement. During long term adsorption in the concentration range of $0.001 \%$ to $0.0001 \%$, phosphatidylcholines in MCT-oil decreased the interfacial tension depending on their fatty acid composition (Fig. 2). The low molecular weight emulsifier with predominantly unsaturated fatty acids, S100, shows a higher interfacial activity in comparison to $90 \mathrm{H}$ with saturated fatty acids in the hydrophobic tail (Fig. 2). The interfacial tension of S100 decreased after $30 \mathrm{~min}$ to $24.5 \pm 0.4 \mathrm{mN} / \mathrm{m}, 18.0 \pm 0.3 \mathrm{mN} / \mathrm{m}$ to $3.9 \pm 0.1 \mathrm{mN} / \mathrm{m}$ with increasing concentration (Fig. 2a). For $90 \mathrm{H}$, the interfacial tension was reduced to $23.8 \pm 0.4$ $\mathrm{mN} / \mathrm{m}, 21.1 \pm 0.3 \mathrm{mN} / \mathrm{m}$ to $20.6 \pm 0.3 \mathrm{mN} / \mathrm{m}$ with increasing concentration (Fig. 2b).

These differences in interfacial tension can be attributed to the interactions and solubility of the low molecular weight emulsifiers in the oil as well as the crystallization behaviour. In general, the interactions of low molecular weight emulsifiers and oil phase as well as solubility of the low molecular weight emulsifier in the oil phase increase with increasing similarity in chain length and saturation [23]. A low molecular weight emulsifier with unsaturated and kinked fatty acids is not strongly integrated in an oil phase with linear nature of saturated fatty acids [22]. Thus, the phosphatidylcholine S100 moves faster and more easily to the interface. In contrast, $90 \mathrm{H}$ contains mainly fatty acids of $\mathrm{C} 16: 0$ and $\mathrm{C} 18: 0$ which can interact strongly with saturated fatty acids $\mathrm{C} 10: 0$ and C8:0 of the MCT-oil. The saturated fatty acids of phosphatidylcholines gets more easily integrated in the tightly packed oil phase with saturated and linear fatty acids [22] and interacts via dispersion forces [21].

Furthermore, the phosphatidylcholine $90 \mathrm{H}$ crystallizes during the cooling period (Fig. 1). If crystallization occurs at the interface and the phosphatidylcholine is covering a high portion of the interface, a network of phosphatidylcholine crystals may induce a change in the drop shape. This was observed as an increase in interfacial tension for the sample $0.001 \% 90 \mathrm{H}$ at $600 \mathrm{~s}$ to $900 \mathrm{~s}$. In previous studies, a rather severe drop shape transformation was observed alongside the use of emulsifiers with long saturated fatty acids during liquid-solid phase transition at the oil/water interface of emulsions [31,32]. It was described that the drop can be deformed by multilayers of emulsifiers at the interface which are inducing a high bending moment which curves the drop shape against the drop surface energy [31,32]. Therefore, the forces of liquid-solid phase transition at interfaces may deform the droplet and affect the analysis of interfacial tension. This effect is measurable from a certain concentration covering a high portion of the droplet interface.

In summary, higher interactions of phosphatidylcholines with the oil phase leads to a lower reduction of the interfacial tension, since occupation of the interface and thus amount of phosphatidylcholine molecules and packing density at the interface are reduced. For long term adsorption of $\beta$-lactoglobulin and phosphatidylcholines, and dilatational 
Fig. 5 Elastic (E') and viscous modulus (E") of frequency (a) and amplitude sweep (b) of $0.01 \% \beta$-lactoglobulin alone or in combination with $0.0001 \%$ phosphatidylcholines $90 \mathrm{H}$ and S100 in MCT-oil. A) amplitude $2 \%$, b) frequency $0.01 \mathrm{~Hz}$. Error bars display the coefficient of variation of the method, letters a-c indicate significant differences between $E$ ' for each frequency (a) or amplitude (b) $(\mathrm{p}<0.05)$.
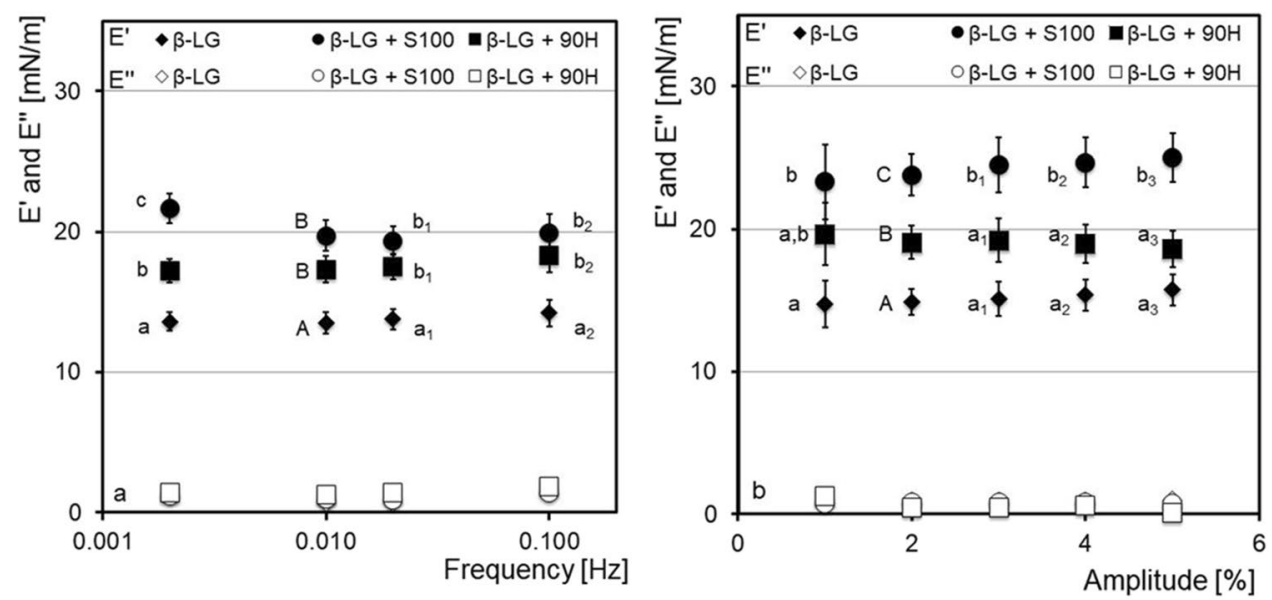

experiments, we have chosen the lowest phosphatidylcholine concentration of $0.0001 \%$.

\section{Long Term Adsorption of $\beta$-Lactoglobulin and Phosphatidylcholines in MCT-Oil}

Interfacial tension measurement on a long-term may be used to verify coexistence of the emulsifying constituents. Thus, the interfacial tension of $\beta$-lactoglobulin with or without presence of the phospholipids $90 \mathrm{H}$ and S100 was analyzed after $3 \mathrm{~h}$ droplet ripening time (Fig. 3). $\beta$-lactoglobulin at $0.01 \%$ lowered the interfacial tension of water against MCToil from $25.4 \pm 0.5 \mathrm{mN} / \mathrm{m}$ to $20.5 \pm 0.4 \mathrm{mN} / \mathrm{m}$. This value is approx. $2.5 \mathrm{mN} / \mathrm{m}$ higher than reported previously for $0.01 \%$ $\beta$-lactoglobulin-film after a droplet ripening time of $1 \mathrm{~h}$ [33]. On one hand this may attributed to differences in time of analysis and inherent properties of the $\beta$-lactoglobulin (e.g. genetic variants, degree of aggregation). On the other hand, the short exposure to elevated temperature immediately after drop generation might have affected the interfacial organization and occupation. It is known, that heat exposure of $\beta$-lactoglobulin located at the interface barely changes the molecular structure of the protein [34], but dynamics of adsorption and interfacial arrangement might be affected by a thermally induced change in mobility.

In both cases $0.0001 \% \mathrm{~S} 100$ or $90 \mathrm{H}$, a combination of $\beta$-lactoglobulin and phosphatidylcholine led to a more pronounced and significant reduction in interfacial tension at the MCT oil-water interface compared to the protein or low molecular weight emulsifiers alone. The interfacial tension decreased to $16.9 \pm 0.3 \mathrm{mN} / \mathrm{m}$ in the $\beta$-lactoglobulin- $\mathrm{S} 100$ system and to $18.5 \pm 0.4 \mathrm{mN} / \mathrm{m}$ in the $\beta$-lactoglobulin- $90 \mathrm{H}$ system. Therefore in both cases, coexistence of the emulsifying constituents can be assumed. This assumption is further confirmed by data on the critical interfacial concentration. For $\beta$-lactoglobulin full interfacial coverage was stated to be slightly above $0.1 \%$ [33], the critical micelle concentration
(CMC) with full interfacial coverage ranged between 0.014 to $0.017 \%$ for several commercial soy-based phosphatidylcholines solubilized in MCT-oil (unpublished data). Therefore, we assume that the concentration of each individual substance was well below the critical concentration for full coverage. The higher and significant reduction of the interfacial tension in the binary system containing S100 compared to $90 \mathrm{H}$ can be attributed to the mechanisms as outlined for the phopspholipids above.

\section{Long Term Adsorption of $\beta$-Lactoglobulin and Phosphatidylcholines in Sunflower Oil}

At a sunflower-oil/water interface, $\beta$-lactoglobulin lowered the interfacial tension from $29.9 \pm 0.5 \mathrm{mN} / \mathrm{m}$ to $24.2 \pm 0.5 \mathrm{mN} / \mathrm{m}$ (Fig. 4). Thus, the decrease in interfacial tension is very similar to the MCT-oil/water interface after $3 \mathrm{~h}$ droplet ripening time (Fig. 3). An impact of oil phase polarity on reduction in interfacial tension of $\beta$-lactoglobulin was described [19] and the same authors showed that more polar lipophilic phases interact with hydrophilic moieties of the $\beta$-lactoglobulin [18]. However, in the present study the difference in molecular structure and polarity between MCT-oil and sunfloweroil might be to small to cause a difference in interfacial behaviour for $\beta$-lactoglobulin.

As hypothesized, addition of the saturated phosphatidylcholine $90 \mathrm{H}$ lowers the interfacial tension significantly and more strongly $(19.3 \pm 0.4 \mathrm{mN} / \mathrm{m})$ at the sunflower oilwater interface than the unsaturated phosphatidylcholine $\mathrm{S} 100(22.8 \pm 0.5 \mathrm{mN} / \mathrm{m})$. The unsaturated phosphatidylcholine S100 interacts with the unsaturated oil [23], which shows a less tightly packed arrangement of fatty acid chains than the saturated oil. The interactions of unsaturated fatty acids of oil phase and low molecular weight emulsifiers are based on very weak $\pi$-interactions of the double bondings or dipersion forces [21, 22] and hinder 
reduction of the interfacial tension. In addition, Hildebrandt et al. (2016) suggested a liquid expanded interfacial layer for an unsaturated phosphatidylcholine in an unsaturated oil and a highly condensed interfacial layer for a saturated phosphatidylcholine in an unsaturated oil [24]. A liquid expanded layer gives more space for each emulsifying constiutent than a condensed layer [35]. Therefore, we assume a higher amount of phosphatidylcholines $90 \mathrm{H}$ fitting on the interface which might reduce the interfacial tension to a higher degree than S100.

\section{Dilatational Rheology of $\beta$-Lactoglobulin and Phosphatidylcholines in MCT-Oil}

During the frequency and amplitude sweep, the elastic modulus of $\beta$-lactoglobulin stays rather constant at approximately $14 \mathrm{mN} / \mathrm{m}$ (Fig. 5). From the amplitude sweep it becomes obvious that the system was in the linear viscoelastic regime and also within the frequency range analyzed no irreversible structural changes in the protein film occurred (Fig. 5). The ratio of the elastic to the viscous modulus is reflected in the phase angle. At an amplitude of $4 \%$ the phase angle amounted to $3.3 \pm 0.9^{\circ}$ for $\beta$-lactoglobulin and thus reflects a viscoelastic behaviour with a high elastic contribution. Upon addition of both phosphatidylcholines, for both sweeps the elastic modulus was higher than for the pure protein film (Fig. 5). E' amounted to approximately $24 \mathrm{mN} / \mathrm{m}$ in the amplitude sweep and $21 \mathrm{~m} / \mathrm{N} / \mathrm{m}$ in the frequency sweep. Values for an interface stabilized through $\beta$-lactoglobulin and $90 \mathrm{H}$ were lower with approximately 19 and $18 \mathrm{mN} / \mathrm{m}$, respectively. The viscous modulus (E") shows no differences between the samples (Fig. 5). The phase angle was lowest in the system with addition of $\mathrm{S} 100\left(1.8 \pm 0.5^{\circ}\right)$ similar to $\beta$-lactoglubulin in the system with addition of $90 \mathrm{H}$ $\left(3.0 \pm 0.8^{\circ}\right)$.

At first the higher values of $E^{\prime}$ in the presence of low molecular weight emulsifiers can be ascribed to the coexistence of the two emulsifying constituents. During compression and expansion, desorption and adsorption of interfacial active molecules to and from the interface occurs [1,9]. During the increase in area within dilatational rheology, due to the non-ideal shape of the drop surface a gradient in interfacial occupation and thus interfacial tension at the interface causes a force to restore the interface with low molecular weight emulsifiers (similar to the Gibbs-Marangoni flow) [9]. Lower values of $\mathrm{E}^{\prime}$ for $90 \mathrm{H}$ compared to S100 (in particular shown in Fig. 5 b) reflect the stronger interactions between the molecule and the oil phase. The high interactions of the lipophilic components decelerate the de- and adsorption processes upon compression/expansion. A crystalline state of the emulsifier was reported to increase the elastic response in comparison to the protein [25-27] based on the rigid reaction of the interface. In the study of
Patino et al. [27] monolaurin increased the elastic modulus to a higher degree than monoolein based on crystallization effects at the interface. However, in the binary system in the present study mobility effects as outlined before obviously dominated the overall behaviour of the system.

The results of the amplitude sweep are depicted as Lissajous-plots (Fig. 6) to gain more information of the interfacial reaction onto expansion and compression. The $\beta$-lactoglobulin-film shows linear and comparably symmetric Lissajous-plots without widening with increasing amplitude. So, the elastic and viscous portions of the film are mostly constant during the amplitude sweep without difference during expansion and compression of the droplet area [36]. The addition of S100 shifts the Lissajous-plot to a slightly steeper angle (Fig. $6 b_{2}$ and $c_{2}$ ). This shift may be attributed to an increase of the change in interfacial tension during oscillation, which may be caused by a reduced number of interactions stabilizing the protein film at the interface. Adsorption of low molecular weight emulsifiers from the bulk did not fully compensate this effect. For $90 \mathrm{H}$, the interfacial film loses elastic portions with increase in oscillation amplitudes which can be seen in the shift to an ellipsoidal shape of Lissajous-plots with increasing amplitude (Fig. $6 \mathrm{~b}_{3}$ and $\mathrm{c}_{3}$ ). This loss is in accordance with the slight loss in the elastic moduli (Fig. 5b) and is attributed to the rigid and crystallized structure at the interface, which tends to break during expansion and compression [27]. The shown data did not indicate non-linearity as discussed in [36].

\section{Dilatational Rheology of $\beta$-Lactoglobulin and Phosphatidylcholines in Sunflower-Oil}

The interfacial characteristics of the $\beta$-lactoglobulin-film in a sunflower-oil/water interface are comparable to the MCT-oil/water interface (Fig. 7). During the amplitude and frequency sweep, the elastic moduli of $\beta$-lactoglobulin stay rather constant (Fig. 7). The addition of phosphatidylcholines increases the elastic moduli, however, the increase is opposite to the data presented for an MCT-oil-water interface in Fig. 5. For S100, the values are increasing with increasing frequency. In the presence of $90 \mathrm{H}$, the values for E' are significantly highest and stay almost constant (Fig. 7). The viscous moduli (E") of the samples are rather similar.

Comparing the data for the two different interfaces, the impact of polarity of the oil phase on the visoelastic behaviour of $\beta$-lactoglobulin [18] was not shown in our study with triaclyglyceride oils with different degree in saturation. As in the case of the adsorption in chapter 3.2 and 3.3, the difference in polarity between MCT-oil and sunflower-oil was too small to cause a difference in interfacial behaviour. The mechanisms responsible for the increase in the elastic moduli in the presence of $\mathrm{S} 100$ and $90 \mathrm{H}$ confirm our previous discussion on the role of interactions with the oil phase and 
Fig. 6 Lissajous-plots of amplitude sweep of $0.01 \%$ $\beta$-lactoglobulin alone $\left(\mathrm{a}_{1}-\mathrm{c}_{1}\right)$ or in combination with $0.0001 \%$ phosphatidylcholines S100 $\left(\mathrm{a}_{2}-\mathrm{c}_{2}\right)$ or $90 \mathrm{H}\left(\mathrm{a}_{3}-\mathrm{c}_{3}\right)$ in MCToil, frequency at $0.01 \mathrm{~Hz}$.
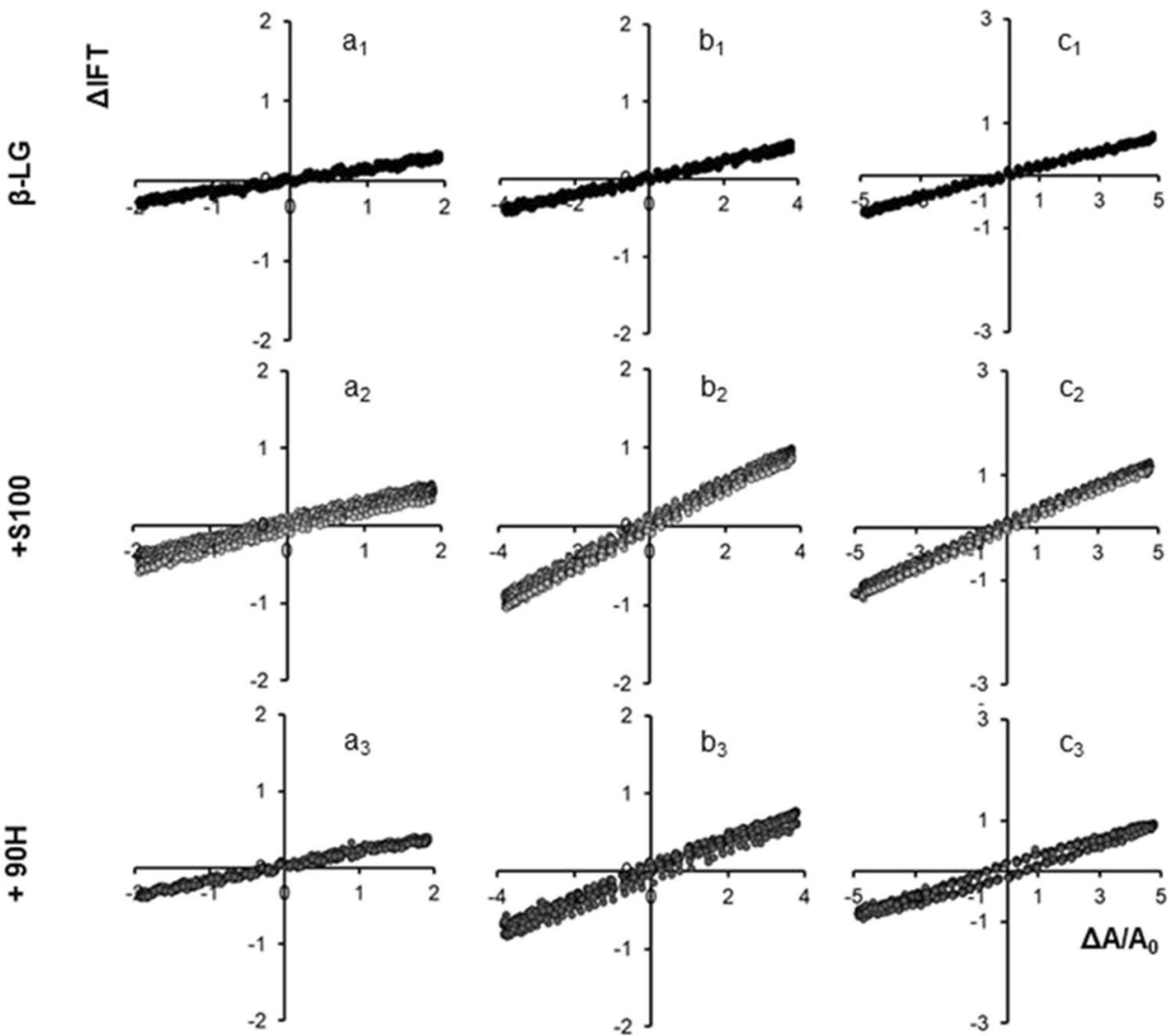

$2 \%$

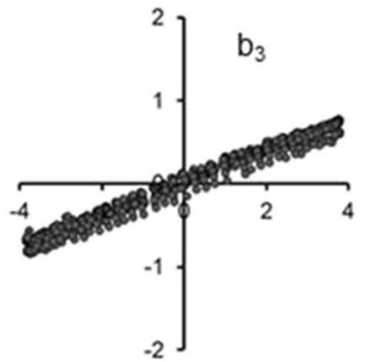

$4 \%$

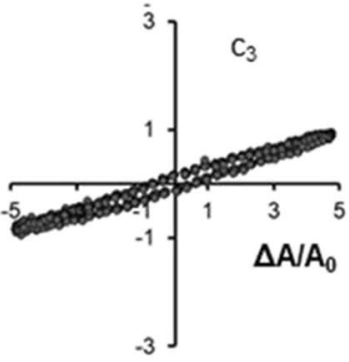

$5 \%$ solubility of the low molecular weight emulsifier. The high solubility of S100 results from higher interactions with the oil phase and a reduced presence of S100 at the interface. Therefore in this setup the elastic modulus is significantly highest for $90 \mathrm{H}$ (Fig. 7) as a consequence of its comparably lower interactions with the oil phase.

The Lissajous-plots show that the $\beta$-lactoglobulinfilm remains elastic without apparent loss in elastic portion with increasing amplitude (Fig. 8). For the addition of S100, Lissajous-plot of the $\beta$-lactoglobulin-film with addition of S100 barely show asymmetric tendencies and is mostly symmetric and linear (Fig. $8 \mathrm{a}_{2}-\mathrm{c}_{2}$ ). The Lissajous-plot of the sample with $90 \mathrm{H}$ shows a steeper angle and is widening with increasing amplitude showing a loss in elastic portion. The loss in elastic portion might be attributed to a rigid and crystallized
Fig. 7 Elastic (E') and viscous modulus (E”) of frequency (a) and amplitude sweep (b) of $0.01 \% \beta$-lactoglobulin alone or in combination with $0.0001 \%$ phosphatidylcholines $90 \mathrm{H}$ and S100 in sunflower-oil. A) amplitude $2 \%$, b) frequency $0.01 \mathrm{~Hz}$. Error bars display the coefficient of variation of the method, letters a-c indicate significant differences between $\mathrm{E}$ ' for each frequency (a) or amplitude (b) $(\mathrm{p}<0.05)$.
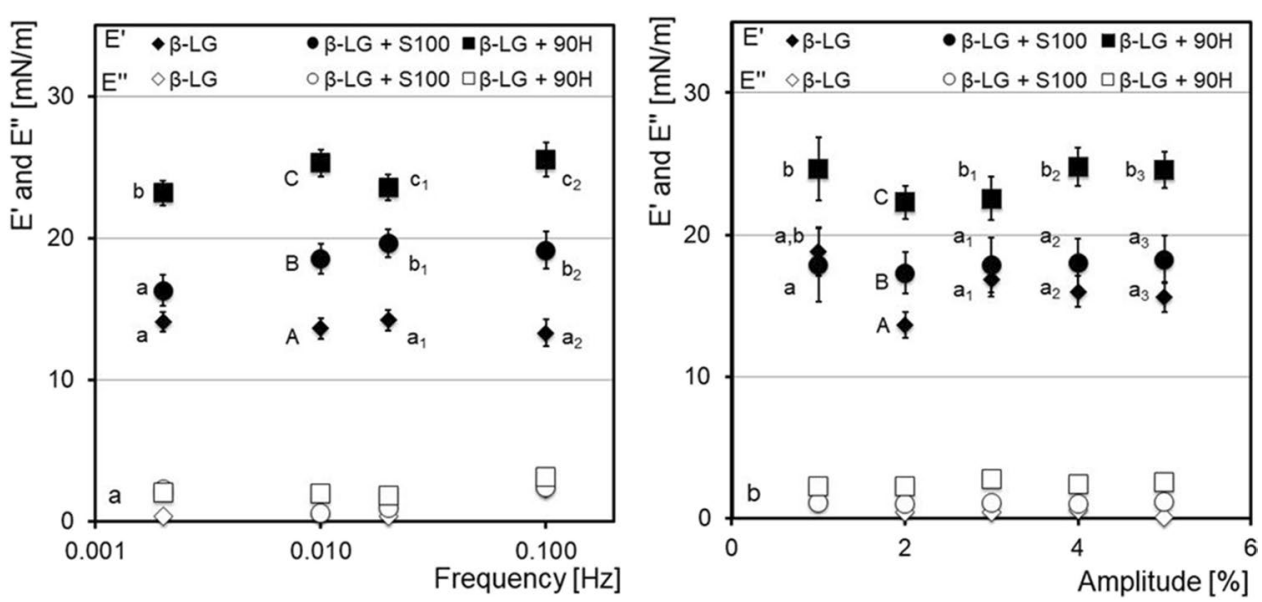
Fig. 8 Lissajous-plots of amplitude sweep of $0.01 \%$ $\beta$-lactoglobulin alone $\left(\mathrm{a}_{1}-\mathrm{c}_{1}\right)$ or in combination with $0.0001 \%$ phosphatidylcholines $\mathrm{S} 100$ $\left(\mathrm{a}_{2}-\mathrm{c}_{2}\right)$ and $90 \mathrm{H}\left(\mathrm{a}_{3}-\mathrm{c}_{3}\right)$ in sunflower-oil, frequency at $0.01 \mathrm{~Hz}$
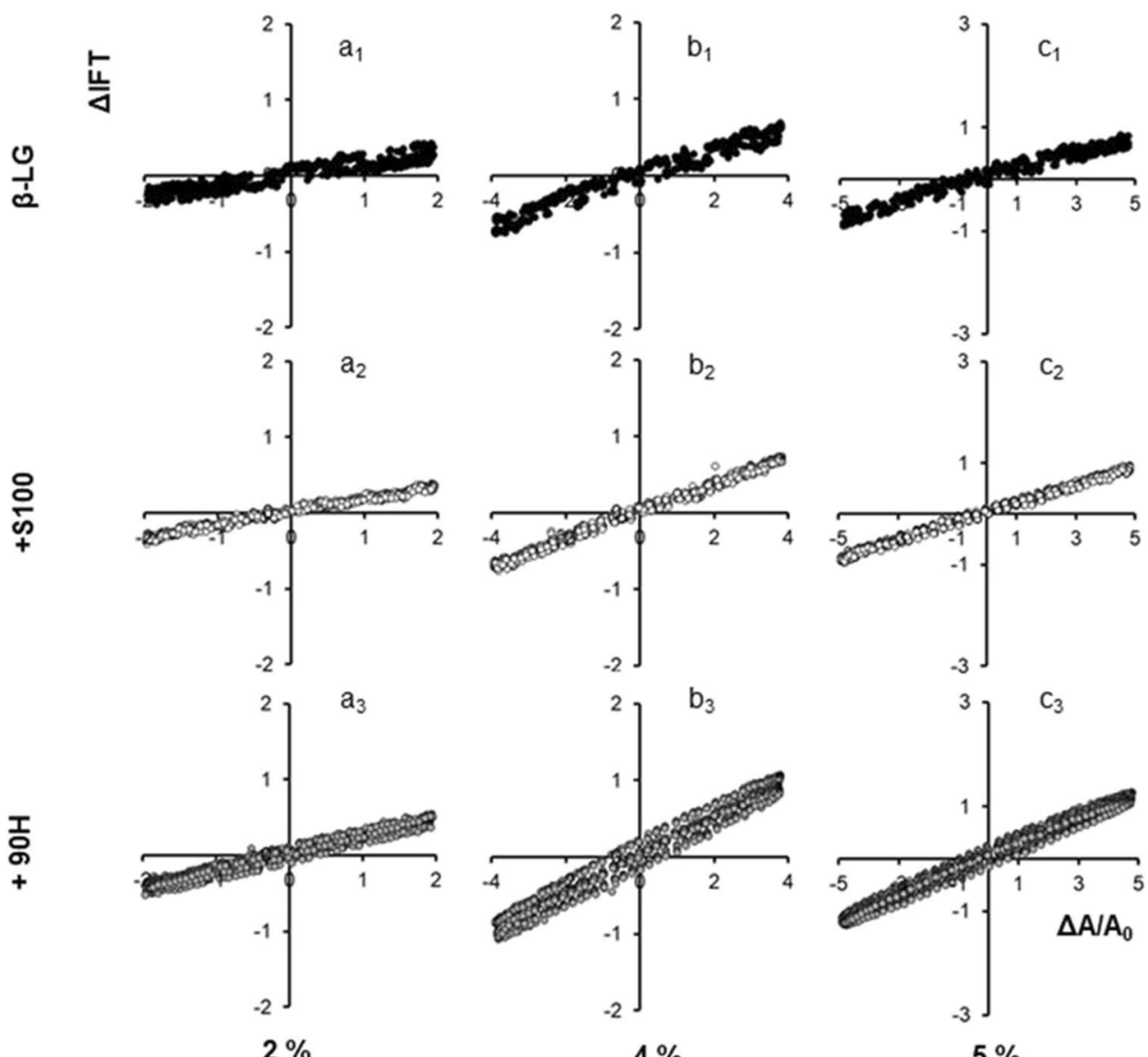

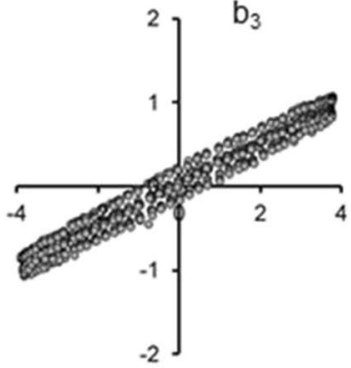

$4 \%$

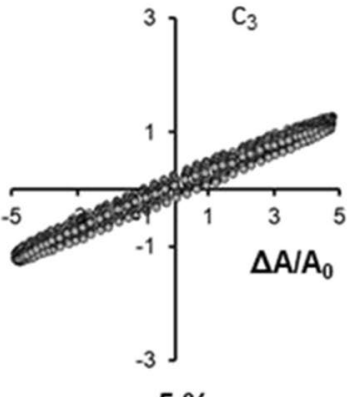

$5 \%$ structure at the interface [27]. That is in accordance with the increase in phase angle for addition with $90 \mathrm{H}$. A similiar effect of loss in elastic portion was observed in the MCT-oil/water interface (Fig. 6). In Fig. 8 these effects are even more pronounced since a template with a high phosphatidylcholine concentration at the interface is formed. Crystals at the interface could be observed with the naked eye in the drop image in the software after measurement.

\section{Conclusion}

The present study analyzed the impact of interactions and solubility of oil phase and low molecular weight emulsifiers depending on saturation of fatty acids on the interfacial properties of $\beta$-lactoglobulin as model protein. The saturation of the oil phase did not affect the interfacial tension or viscoelasticity of $\beta$-lactoglobulin-films. In contrast, the saturation of the oil phase had a large impact on interfacial characteristics of phosphatidylcholines. If a phosphatidylcholine is highly soluble in the oil phase, the interactions with the oil phase are reducing its potential to lower the interfacial tension in comparison to a phosphatidylcholine with low solubility. The interactions result in a lower interfacial occupation and higher interfacial tension. In a system with $\beta$-lactoglobulin, the interfacial tension is higher if high interactions of phosphatidylcholine and oil phase exist. The characteristics and stability of the interfacial film of $\beta$-lactoglobulin-phosphatidylcholine are further affected by interfacial adsorption during changes in interfacial area, and crystallization of low molecular weight emulsifiers. Future research should also cover interfacial rheology in the nonlinear regime to get a deeper inside into stress-response of the mixed films.

However, it is already obvious that all these effects are of importance for the application in food or pharmaceutical products. Therefore, the choice of saturation of fatty acids of oil phase and low molecular weight emulsifier is rather important. For instance, a solid template at the interface could hinder oil droplet destabilization by oil crystallization if the emulsion is exposed to temperatures below the oil crystallization temperature during storage time $[37,38]$. The transferability to other low molecular weight emulsifiers like mono- and diglycerides or citrem or oil phases like alkanes needs to be analyzed. The upcoming results will help to systematically understand the impact of interactions of low molecular weight emulsifiers and oil phase on interfacial properties of proteins. 
Acknowledgements The authors gratefully acknowledge the skillful lab-work of Sarah Gleisenberg ( $\beta$-lactoglobulin-isolation) and Julia Keppler (analysis of $\beta$-lactoglobulin quantification). Furthermore, Monika Brückner-Gühmann and Helena Kieserling helped with their skillful proofreading of the manuscript. Simon Heiden enriched the manuscript by illustrating the $\beta$-LG molecule. Theresia Heiden-Hecht thanks Daniela and Michael Schäfer for valuable discussions.

Authors' Contributions T. Heiden-Hecht: conceptualization, methodology, validation, investigation, writing-original draft, visualization, writing-review \& editing.

S. Drusch: supervision, writing - review \& editing.

Funding Open Access funding enabled and organized by Projekt DEAL.

\section{Declarations}

Conflict of Interest The authors declare that they have no known competing financial interests or personal relationships that could have appeared to influence the work reported in this paper.

Open Access This article is licensed under a Creative Commons Attribution 4.0 International License, which permits use, sharing, adaptation, distribution and reproduction in any medium or format, as long as you give appropriate credit to the original author(s) and the source, provide a link to the Creative Commons licence, and indicate if changes were made. The images or other third party material in this article are included in the article's Creative Commons licence, unless indicated otherwise in a credit line to the material. If material is not included in the article's Creative Commons licence and your intended use is not permitted by statutory regulation or exceeds the permitted use, you will need to obtain permission directly from the copyright holder. To view a copy of this licence, visit http://creativecommons.org/licenses/by/4.0/.

\section{References}

1. P. Wilde, A. Mackie, F. Husband, P. Gunning, V. Morris, Adv. Colloid Interf. Sci. 108-109, 63 (2004)

2. R.S.H. Lam, M.T. Nickerson, Food Chem. 141, 975 (2013)

3. E. Dickinson, Food Hydrocoll. 25, 1966 (2011)

4. L.A. Pugnaloni, E. Dickinson, R. Ettelaie, A.R. Macke, P.J. Wilde, Adv. Colloid Interf. Sci. 107, 27 (2004)

5. C. Vega, Y.H. Roos, J. Dairy Sci. 89, 383 (2006)

6. R.C. Pasquali, N. Sacco, C. Bregni, Lat. Am. J. Pharm. 28, 313 (2009)

7. D.J. McClements, S. Mahdi Jafari, Adv. Colloid Interf. Sci. 251, 55 (2018)

8. R. J. (Eds) Whitehurst, Emulsifiers in Food Technology (Blackwell Publishing Ltd, Oxford, UK, 2004)

9. B.S. Murray, E. Dickinson, Food Sci. Technol. Int. 2, 131 (1996)

10. M.A. Bos, T. van Vliet, Adv. Colloid Interf. Sci. 91, 437 (2001)

11. A. Dan, G. Gochev, J. Krägel, E.V. Aksenenko, V.B. Fainerman, R. Miller, Curr. Opin. Colloid Interface Sci. 18, 302 (2013)
12. C. Kotsmar, V. Pradines, V.S. Alahverdjieva, E.V. Aksenenko, V.B. Fainerman, V.I. Kovalchuk, J. Krägel, M.E. Leser, B.A. Noskov, R. Miller, Adv. Colloid Interf. Sci. 150, 41 (2009)

13. L. Cui, E.A. Decker, J. Sci. Food Agric. 96, 18 (2016)

14. V. Mitropoulos, A. Mütze, P. Fischer, Adv. Colloid Interf. Sci. 206, 195 (2014)

15. R. Wüstneck, B. Moser, G. Muschiolik, Colloids Surfaces B Biointerfaces 15, 263 (1999)

16. E.H. Lucassen-Reynders, J. Benjamins, V.B. Fainerman, Curr. Opin. Colloid Interface Sci. 15, 264 (2010)

17. S. Böttcher, J.K. Keppler, S. Drusch, Colloids Surfaces A Physicochem. Eng. Asp. 518, 46 (2017)

18. J. Bergfreund, P. Bertsch, S. Kuster, P. Fischer, Langmuir 34, 4929 (2018)

19. J. Bergfreund, P. Bertsch, P. Fischer, J. Colloid Interface Sci. 584, 411 (2021)

20. J. Bergfreund, S. Siegenthaler, V. Lutz-Bueno, P. Bertsch, P. Fischer, Langmuir 37, 6722 (2021)

21. P. Walstra, Physical Chemistry of Foods (Marcel Dekker, Inc, 2003)

22. H. D. Belitz, W. Grosch, and P. Schieberle, Food Chemistry (Springer Berlin Heidelberg, 2009)

23. N. Garti and J. Yano, in Cryst. Process. Fats Lipid Syst., edited by N. Garti and K. Sato (Marcel Dekker, Inc; New York, USA; Basel, Switzerland, 2001), p. $211 \mathrm{ff}$

24. E. Hildebrandt, A. Dessy, J.H. Sommerling, G. Guthausen, H. Nirschl, G. Leneweit, Langmuir 32, 5821 (2016)

25. M. Golding, A. Sein, Food Hydrocoll. 18, 451 (2004)

26. C.C. Sánchez, J.M. Rodríguez Patino, Langmuir 20, 6327 (2004)

27. J.M. Rodríguez Patino, M.R. Rodríguez Nino, C. Carrera Sánchez, J.M. Navarro García, G.R. Rodríguez Mateo, M. Cejudo Fernández, Colloids Surfaces B Biointerfaces 21, 87 (2001)

28. J.K. Keppler, F.D. Sönnichsen, P.-C. Lorenzen, K. Schwarz, Biochim. Biophys. Acta 1844, 1083 (2014)

29. H. Schestkowa, T. Wollborn, A. Westphal, A.M. Wagemans, U. Fritsching, S. Drusch, J. Colloid Interface Sci. 536, 300 (2019)

30. E.H. Lucassen-Reynders, Food Struct. 12, 1 (1993)

31. N. Denkov, S. Tcholakova, I. Lesov, D. Cholakova, S.K. Smoukov, Nature 528, 392 (2015)

32. N. Denkov, S. Tcholakova, D. Cholakova, Curr. Opinon Colloid Interface Sci. 44, 32 (2019)

33. H. Schestkowa, S. Drusch, A.M. Wagemans, Food Chem. 302, 125349 (2020)

34. J. Zhai, A.J. Miles, L.K. Pattenden, T.-H. Lee, M.A. Augustin, B.A. Wallace, M.-I. Aguilar, T.J. Wooster, Biomacromolecules 11, 2136 (2010)

35. R. Pichot, R.L. Watson, I.T. Norton, Int. J. Mol. Sci. 14, 11767 (2013)

36. L.M.C. Sagis, P. Fischer, Curr. Opin. Colloid Interface Sci. 19, $520(2014)$

37. L. Goibier, S. Lecomte, F. Leal-Calderon, C. Faure, J. Colloid Interface Sci. 500, 304 (2017)

38. E. Fredrick, B. Heyman, K. Moens, S. Fischer, T. Verwijlen, P. Moldenaers, P. Van der Meeren, K. Dewettinck, Food Res. Int. 51, 936 (2013)

Publisher's Note Springer Nature remains neutral with regard to jurisdictional claims in published maps and institutional affiliations. 